\title{
Comparação da Qualidade do Ar em Localidades Industrial e Urbana
}

\author{
Raphael Perciliano da Silva de Almeidal; Thainá de Carvalho e Souzal; Simone Lorena Quiterio de \\ Souza ${ }^{2}$; Eduardo Monteiro Martins'; Sergio Machado Corrêall ${ }^{l}$
}

\raphael@ecoteceng.com.br

1. Universidade do Estado do Rio de Janeiro, RJ. 2. Instituto Federal de Educação, Ciência e Tecnologia.

Histórico do Artigo:

Recebido em: 22 de maio de 2019 Aceito em: 02 de setembro de 2019 Publicado em: 28 de dezembro de 2019

Resumo: Este estudo compara a qualidade do ar de duas localidades da Região Metropolitana do Rio de Janeiro, a saber: uma área predominantemente impactada pelas emissões veiculares na Taquara (TQ), Jacarepaguá e a outra pelas emissões industriais em Jardim Primavera (JP), Duque de Caxias. Os dados utilizados correspondem às médias horárias entre 2014 a 2016 para óxidos de nitrogênio (N0x, N02 e N0), ozônio (03), monóxido de carbono (C0), dióxido de enxofre (S02) e material particulado (MP10), além de dados meteorológicos. Para interpretar os resultados utilizou-se a Linguagem R. A razão calculada entre as duas localidades para os valores de concentração mediana (JP: 10,7 ppb; TQ: 5,4 ppb) e máxima (JP: 197 ppb; TQ: 146 ppb) para 03 foi de 1,98 e 1,35, respectivamente. Foi apresentada a importância da utilização de software estatístico para o manuseio e tratamento dos dados e apresentação de resultados provenientes de estações de qualidade do ar.

Palavras-chave: Emissões Atmosféricas, Estatística Multivariada, Poluição Atmosférica, Meteorologia.

\section{Comparison of Air Quality in Industrial and Urban Locations}

\begin{abstract}
This study compares the air quality of two locations in the Metropolitan Region of Rio de Janeiro, namely: an area predominantly impacted by vehicular emissions in Taquara (TQ), Jacarepaguá and the other by industrial emissions in Jardim Primavera (JP), Duque de Caxias. The data used correspond to the averages between 2014 and 2016 for nitrogen oxides (NOx, N02 and N0), ozone (03), carbon monoxide (CO), sulfur dioxide (S02) and particulate matter (MP10) meteorological data. In order to interpret the results, the language R. The calculated ratio between the two locations for the values of median (JP: 10.7 ppb; TQ: 5.4 ppb) and maximum (JP: 197 ppb; TQ: 146 ppb) for 03 was 1.98 and 1.35, respectively. The importance of the use of statistical software for the handling and processing of data and the presentation of results from air quality stations was presented.
\end{abstract}

Keywords: Atmospheric emissions, Multivariate Statistics, Atmospheric pollution, Meteorology.

\section{Comparación de la Calidad del Aire en Localidades Industrial y Urbana}

Resumen: Este estudio compara la calidad del aire de dos localidades de la Región Metropolitana de Río de Janeiro, a saber: un área predominantemente impactada por las emisiones vehiculares en Taquara (TQ), Jacarepaguá y la otra por las emisiones industriales en Jardim Primavera (JP), Duque de Caxias. Los datos utilizados corresponden a los promedios horarios entre 2014 a 2016 para óxidos de nitrógeno (NOx, N02 y N0), ozono (03), monóxido de carbono (C0), dióxido de azufre (S02) y material particulado (MP10) datos meteorológicos. Para interpretar los resultados se utilizó el Lenguaje R. La razón calculada entre las dos localidades para los valores de concentración mediana (JP: 10,7 ppb; TQ: 5,4 ppb) y máxima (JP: 197 ppb; TQ: 146 ppb) para 03 fue de 1,98 y 1,35, respectivamente. Se muestra la importancia del uso de software estadístico para el manejo y tratamiento de los datos y la presentación de resultados procedentes de estaciones de calidad del aire.

Palabras clave: Emisiones atmosféricas, Estadística Multivaria, Contaminación atmosférica, Meteorología. 


\section{INTRODUÇ̃̃o}

0 estudo da qualidade do ar de grandes cidades envolve diferentes aspectos, como as emissões, as transformações físico-químicas e meteorologia, bem como as influências topográficas, o que torna esse tipo de estudo altamente não linear (MARQUES, 2017; MUNIR; CHEN; ROPKINS, 2013; MONTEIR0 et al., 2012; CORRÊA; MARTINS; ARBILLA, 2003). As atividades exercidas pela humanidade, desde 0 início da era industrial, promoveram benefícios econômicos e sociais, entretanto provocaram grandes alterações na composição química da troposfera, através da emissão de poluentes, especialmente nos ambientes urbanos e industriais (ORLANDO et al., 2010).

Considerando a poluição do ar como um dos principais problemas em áreas urbanas e industriais, é importante buscar a forma mais adequada para o crescimento industrial e da frota veicular, viabilizando o desenvolvimento da economia e também minimizando os impactos para que a qualidade do ar não seja afetada significativamente (MAIA et al., 2014; WANG et al., 2010). Entre os principais impactos negativos, os poluentes atmosféricos, têm maior impacto na saúde humana quando atingem o sistema respiratório, gerando ou potencializando os seus efeitos deletérios como doenças respiratórias, e problemas neurológicos (MUNIR; CHEN; ROPKINS, 2013).

0 acompanhamento da poluição atmosférica em escala local ou regional normalmente é realizado pela rede de monitoramento da qualidade do ar. Esta rede constitui uma ferramenta útil para a segurança da saúde humana e do meio ambiente, permitindo analisar o benefício de ações de saneamento e predeterminar intervenções planejadas quando as concentrações estiverem acima dos padrões de qualidade do ar estabelecido pela legislação (VITALI et al., 2016; MOREIRA; TIRABASSI, 2006).

Diante disso, as agências ambientais efetuam o monitoramento da qualidade do ar, cabendo destacar que cada localidade denota características próprias e diferentes, desde 0 perfil socioeconômico, até o volume populacional, fontes de emissão, aspectos meteorológicos e topográficos distintos (MONKS et al., 2015). 
Desta forma, esse trabalho tem como objetivo comparar a qualidade do ar no período entre 2014 a 2016 de duas estações da qualidade do ar com perfis de emissão diferentes, uma área predominantemente impactada pelas emissões veiculares na Taquara (TQ), Jacarepaguá e a outra pelas emissões industriais em Jardim Primavera (JP), Duque de Caxias. Com os dados obtidos através das estações automáticas da qualidade do ar do INEA, foi utilizada a Linguagem R para o estudo estatístico descritivo e multivariado dos dados.

\section{MATERIAL E MÉTODOS}

A atual rede de monitoramento da qualidade do ar do INEA é composta por 58 Estações de Monitoramento da Qualidade do Ar (EMQAr.) automáticas, que realizam determinações de gases (óxidos de nitrogênio (NOx), monóxido de carbono (C0), dióxido de enxofre (S02), ozônio(03), hidrocarbonetos (HC)) e material particulado (MP). Sendo 116 amostradores para a rede semiautomática, capazes de realizar o monitoramento das concentrações de material particulado total (PTS), inalável (PI) ou respirável (MP2.5), por 24 horas ininterruptas, de 6 em 6 dias. As estações que compõem a rede de monitoramento da qualidade do ar do Estado do Rio de Janeiro estão distribuídas na Região Metropolitana do Rio de Janeiro (RMRJ), na Região Médio Paraíba (RMP) e na Região Norte Fluminense (RNF) (INEA, 2015).

A rede automática processa os dados de concentração dos parâmetros de qualidade do ar e meteorologia na forma de médias horárias, online e em tempo real. Estas médias são transmitidas para a central de telemetria e armazenadas em servidor de banco de dados, onde posteriormente passam por processo de validação técnica periódica para que sejam disponibilizadas através de boletins diários no endereço eletrônico do INEA (INEA, 2015).

A representatividade dos dados é calculada considerando os critérios de validação dos dados utilizados pelo órgão ambiental. Para as médias horárias $75 \%$ das medidas válidas em 1 hora e para médias diárias o critério de validação foi 75 \% das medidas válidas em 24 horas. Todos os dados utilizados neste trabalho já passaram pelo processo de validação aplicado pelo órgão ambiental (INEA, 2015).

A Região Metropolitana do Rio de Janeiro (RMRJ) é composta por 21 municípios que concentram 74,7 \% da população do Estado (CEPERJ, 2018), com 68 \% do PIB (Produto Interno 
Bruto), estadual e com um Índice de Desenvolvimento Humano Municipal (IDHM), de 0,799 (IBGE, 2018). Diante do aumento contínuo da população, aparecimento de novas unidades industriais e aumento expressivo do número de automóveis, vários são os desafios para manter a qualidade do ar dentro dos parâmetros regulatórios vigentes, desta forma, a poluição atmosférica continua a crescer ano após ano (GERALDIN0 et al., 2017; UEDA, 2010).

Entre as estações de monitoramento da qualidade do ar (EMQAr) da RMRJ foram escolhidas as estações da Taquara (TQ) localizada no bairro de Jacarepaguá por constituir uma estação com emissões predominantemente veiculares levando em consideração os poluentes monitorados pela estação e a quantidade de dados validados e disponibilizados para o período escolhido em detrimento a outras estações com o mesmo perfil. E, a estação de Jardim Primavera (JP) em Duque de Caxias para representar a qualidade do ar com forte influência de áreas industriais, por apresentar a maior quantidade de dados existentes e por conter todos os poluentes legislados monitorados. A estação Jardim Primavera (JP) está distante aproximadamente 5,5 km do Polo Petroquímico existente na cidade de Duque de Caxias.

0 período escolhido abrangeu dois eventos de grande porte como a Copa do Mundo em 2014, onde o Rio de Janeiro foi Cidade-Sede e as Olimpíadas de 2016 sedeada no Rio de Janeiro. Ambos os eventos trouxeram grandes alterações urbanísticas para a cidade que promoveram benefícios à metrópole, como a implantação dos corredores de ônibus expresso.

A EMQAr da Taquara localiza-se dentro do estacionamento do laboratório de uma grande indústria farmacêutica na região geográfica da Baixada de Jacarepaguá (22.934657 S, 43.371727 0), zona oeste do Rio de Janeiro. Fica a poucos metros das estações do BRT Merck e Santa Efigênia. Com população estimada de 102.126 mil habitantes e área territorial de aproximadamente 1.320,66 hectares o bairro é densamente residencial e urbanizado (IBGE, 2018).

A EMQAAr de Jardim Primavera fica alocada nas imediações do Arco Metropolitano, dentro do pátio da Polícia Rodoviária Federal, coordenadas 22.674612 S e 43.285100 0, nas proximidades do polo petroquímico situado em Campos Elíseos. A localização de cada estação pode ser visualizada na Figura 1. 


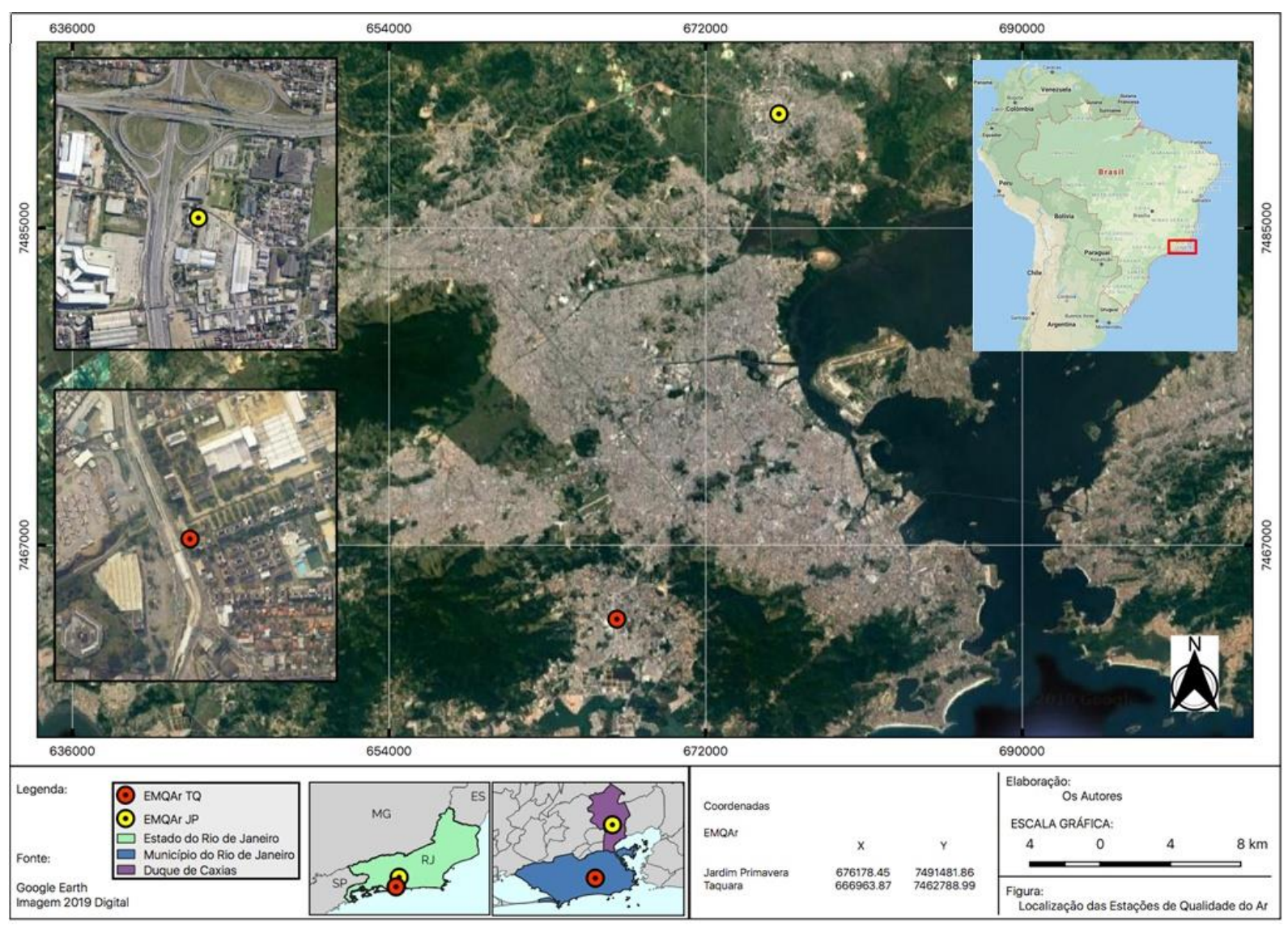

Figura 1 - Localização das estações de monitoramento da qualidade do ar na Taquara e em Jardim Primavera.

Fonte: 0s Autores.

Os dados iniciais coletados totalizam três anos de dados válidos para os poluentes: NOX, N0, N02, 03, S02, C0, MP10, e ainda os parâmetros meteorológicos de velocidade do vento (ws), direção do vento (wd) e temperatura (T). A quantidade de observações originais foram 26.270 casos de médias horárias para 10 variáveis da estação da Taquara (TQ) e 26.175 casos de médias horárias para 10 variáveis da estação de Jardim Primavera (JP). Não foram removidos dados com chuva, fim de semana e feriados.

Para a análise estatística dos dados monitorados nas estações automáticas foi utilizado a Linguagem R (R CORE TEAM, 2017) que é de código aberto e fornece uma grande variedade ferramentas estatísticas. Toda a programação foi realizada através do ambiente RStudio integrado ao R. A escolha por tal código computacional levou em conta aspectos como, existência de pacote voltado para qualidade do ar e a sua livre distribuição. 0 principal pacote utilizado foi o OpenAir. Dentro deste pacote, foram exploradas as ferramentas Timeplot, windRose, polarAnnulus, polarPlot, CorrPlot e timeVariation. 


\section{RESULTAD0S E DISCUSSÃ0}

A evolução temporal das médias horárias das concentrações das EMQAr da TQ e JP estão apresentadas na Figura 2 (2.1 e 2.2 respectivamente), onde os sombreamentos mostram os intervalos de confiança de $95 \%$ da média. Estes são resultados das tendências e da variabilidade cíclica das concentrações de poluentes observadas nas duas estações.
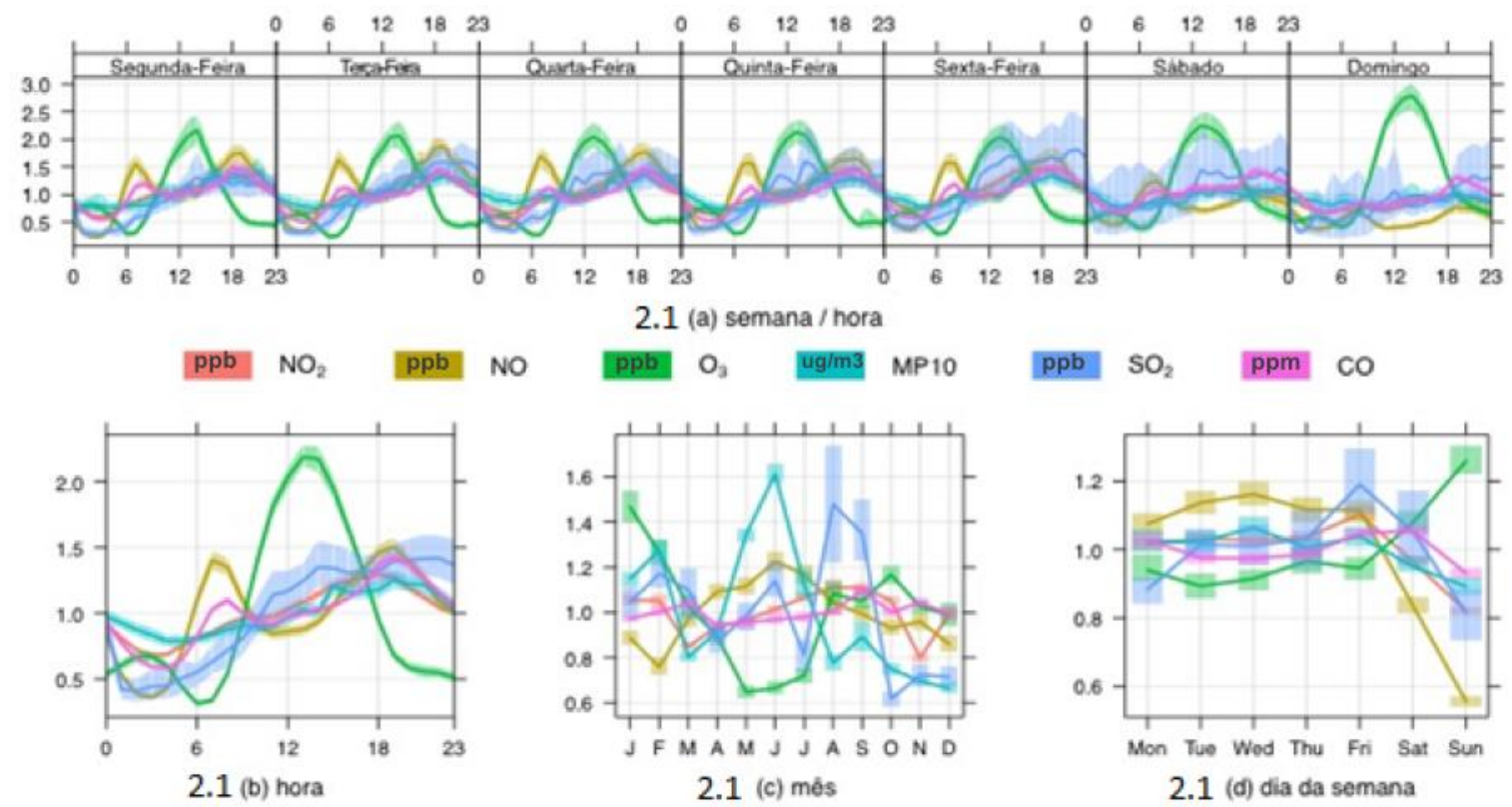

ppb $\mathrm{SO}_{2} \quad \mathrm{ppm} \mathrm{CO}$

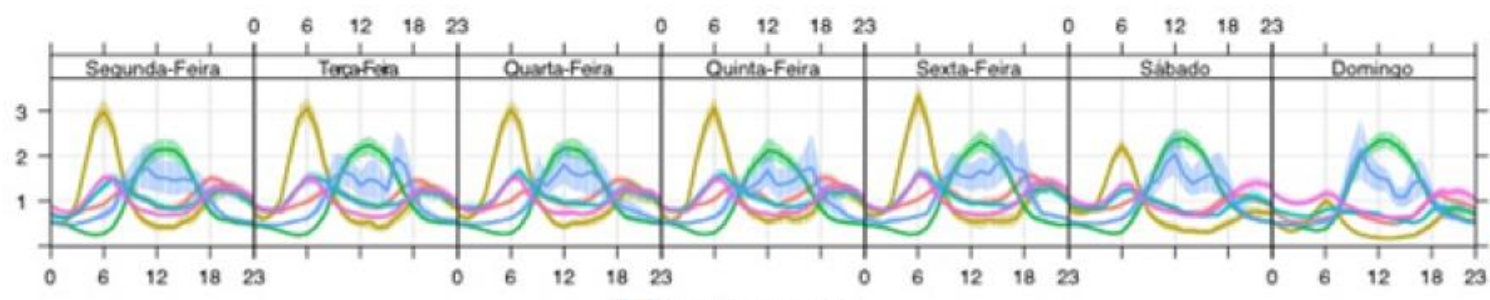

2.2 (a) semana / hora

ppb $\mathrm{NO}_{2} \quad \mathrm{ppb}$ NO
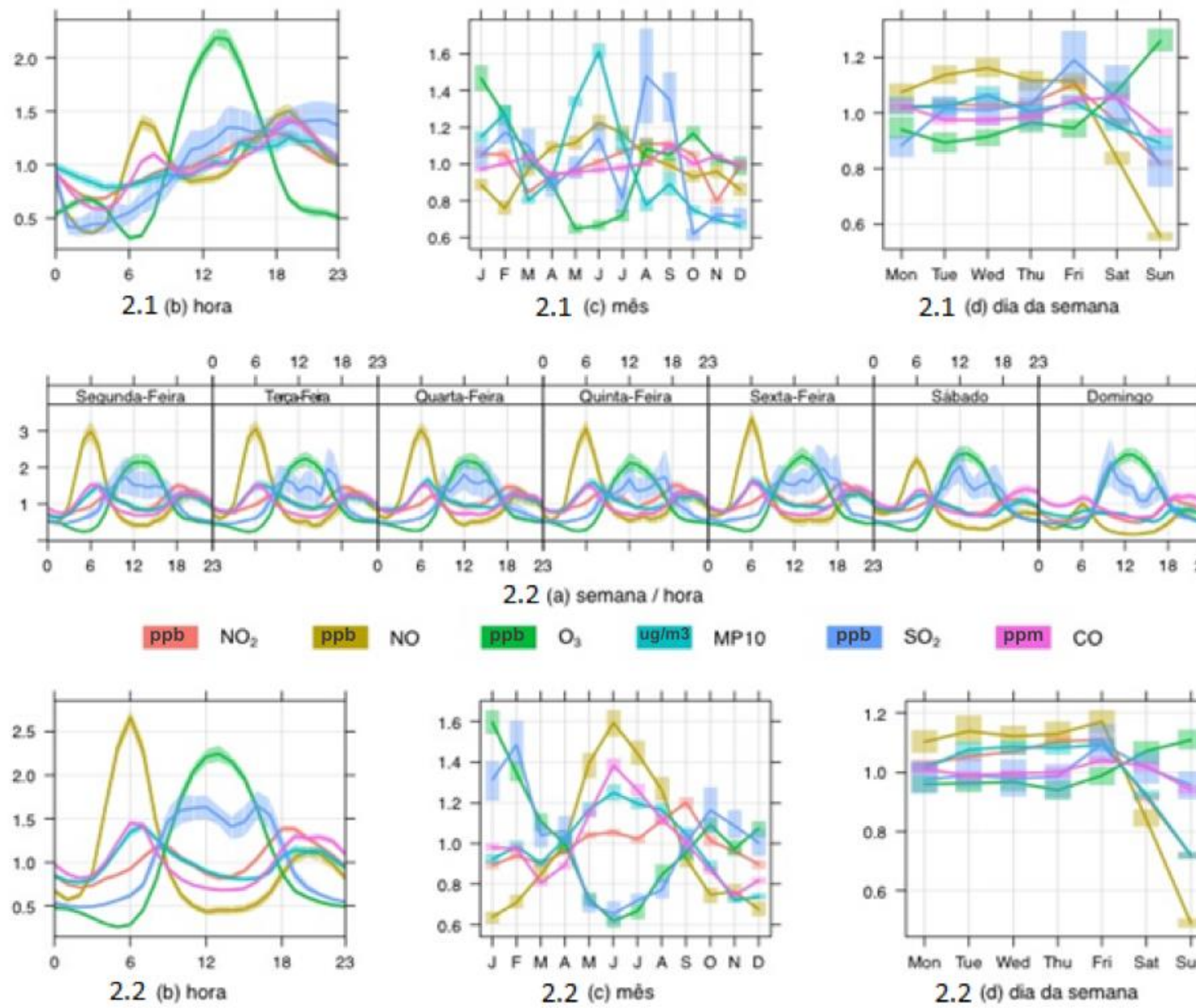

2.1 (d) dia da semana

$\mathrm{ppb} \mathrm{SO}_{2} \quad$ ppm $\mathrm{CO}$

Figura 2 - Perfis de concentrações médias dos poluentes na EMQAr TQ (2a) e JP (2b) entre 20142016.

Fonte: Os autores.

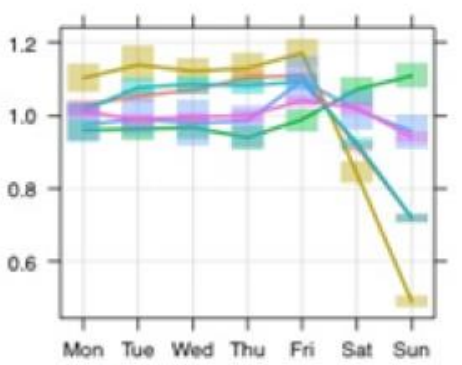

2.2 (d) dia da semana 
Analisando os dias da semana na TQ (Figura 2.la), os resultados indicam que o N02 e MP10 são provenientes da mesma fonte emissora, pois os perfis de ambos são semelhantes em todos os períodos do dia e dias da semana, mostrando a relação entre estes dois poluentes. Segundo Carslaw, (2015) isso ocorre devido às emissões de poluentes oriundos de veículos rodoviários que tendem a seguir padrões muito regulares, tanto de horário, diário quanto semanal.

Os resultados de N0 apresentaram dois picos, um no início da manhã, caracterizado pelo rush matinal, onde o fluxo de automóveis aumenta consideravelmente neste período (GERALDIN0 et al., 2017) e outro no final da tarde para noite, influenciado pelo retorno destes veículos ao seu ponto original.

Analisando a periodicidade mensal, os meses centrais do calendário, relacionado ao período de inverno, apresentam as maiores concentrações para N02, N0 e MP10 e as menores concentrações para o 03 em ambas as EMQAr. Filip Soporan et al., (2015); Michanowicz et al., (2016); Wang et al., (2010) relatam que no inverno o fenômeno das inversões térmicas, em condições de extrema estabilidade, contribui negativamente para a dispersão dos poluentes. Este fenômeno ocorre quando uma camada de ar quente ficando em cima de uma camada de ar frio, bloqueia os movimentos verticais de convecção. Uma vez que no inverno esta camada atinge baixas altitudes, a dispersão dos gases nas correntes verticais é dificultada, o que explicaria as maiores concentrações de N02, N0 e MP10 no inverno. Quanto as baixas concentrações de 03 neste mesmo período, deve-se ao fato da incidência solar diminuir (VALOTTO; VARIN, 2016).

Na EMQAr JP, os perfis de concentração de N0 apresentaram comportamento um pouco distintos aos da EMQAr TQ. Analisando em função das horas do dia, apesar das concentrações de N0 também terem apresentado dois picos, o pico da manhã é muito maior que o pico da tarde, como pode ser visto nas Figuras 2.la e 2.1b. Isto deve ser influenciado pela proximidade da estação JP da Rodovia Washington Luiz, BR-040/RJ, onde o trafego de veículos pesados é muito mais intenso e representativo e, por esta rodovia apresentar diversas faixas de rolagem, o que aumenta a quantidade de veículos transitando. Ao lado e nas proximidades da estação JP existem locais de movimentação de caminhões, como base logísticas, estacionamentos e paradas de caminhões. 
$00_{3}$, com picos no período da tarde, manteve um comportamento praticamente idêntico em todos os dias da semana tanto em TQ apresentados nas Figuras 2.la e 2.1d quanto em JP apresentados nas Figuras 2.2a e 2.2d, com maiores concentrações nos finais de semana, porém em diferentes intensidades. 0 oposto foi observado para o $\mathrm{NO}_{2}$, $\mathrm{NO}$ e $\mathrm{MP}_{10}$ que tiveram suas concentrações reduzidas no sábado e no domingo, principalmente. Algumas causas possíveis podem justificar estas diferenças aos finais de semana, como por exemplo, a diferença entre as emissões do veículo entre dias úteis e fins de semana. As duas estações possuem tanto fontes móveis (veículos) quanto fontes fixas (indústrias) ao seu redor, porém na JP as emissões destes dois tipos de fontes são mais acentuadas em relação a TQ. As maiores concentrações de $0_{3}$ aos finais de semana ocorreram na JP, porque baixas concentrações de NO resultaram em um baixo consumo de 03 como mostrado nas Reações 1 a 3 (MARTINS et al., 2015).

$$
\begin{aligned}
& \mathrm{NO}_{2}+\mathrm{h} v \rightarrow \mathrm{NO}+\mathrm{O}\left({ }^{3} \mathrm{P}\right)(<420 \mathrm{~nm}) \\
& \mathrm{O}\left({ }^{3} \mathrm{P}\right)+\mathrm{O}_{2}+\mathrm{M} \rightarrow \mathrm{O}_{3} \\
& \mathrm{O}_{3}+\mathrm{NO} \rightarrow \mathrm{NO}_{2}+\mathrm{O}_{2}
\end{aligned}
$$

As Figuras 2.1b e 2.2b apresentam a evolução ao longo de 24 horas das concentrações médias horárias de todo o triênio para TQ e JP. É possível observar que que após as 5 h, a concentração de NO tende a aumentar devido ao tráfego veicular e o $0_{3}$ remanescente do dia anterior é então consumido para formação de $\mathrm{NO}_{2}$. Em seguida, entre 6 e 8 h, com o início da radiação solar, o $\mathrm{NO}_{2}$ é fotolisado para a formar de $0_{3}$, aumentando os níveis de $0_{3}$ e diminuindo os níveis de $\mathrm{NO}_{2}$. Esse processo atinge sua máxima produção de $0_{3}$ após o máximo da radiação solar, pois é quando a taxa de fotólise da reação 1 é máxima. Poucas horas depois da taxa máxima de fotólise da reação 1 , novamente os níveis de $\mathrm{NO}_{2}$ voltam a crescer, uma vez que a taxa de formação de $0_{3}$ decai, devido à redução da radiação solar (ATKINSON, 2000). Na ausência de luz solar, os processos de consumo de $0_{3}$ vão prevalecer e os processos de formação serão inexistentes, as concentrações de $\mathrm{O}_{3}$ são menores e as concentrações de $\mathrm{NO}_{2}$ maiores (IEMA, 2014).

A técnica de normalização facilitou a interpretação dos resultados de concentração de $\mathrm{SO}_{2}$ e $\mathrm{CO}$ e permitiu avaliar as possíveis correlações destes com os locais de estudo. Para o CO, há um aumento pronunciado nas concentrações durante os dois períodos de rush na TQ e em 
JP. A outra diferença importante ocorreu aos domingos, quando as concentrações de C0 em ambas as estações foram relativamente mais altas que o $\mathrm{NO}_{2}$ e NO. Este cenário relaciona-se com as análises feitas por Carslaw, (2015) na estrada Marylebone em Londres, que verificou que o fluxo de carros (principalmente gasolina) não muda muito em relação aos dias da semana, mas o fluxo de veículos pesados como, por exemplo, caminhões e o BRT (veículos a diesel) são muito menores aos domingos.

As concentrações de $\mathrm{SO}_{2}$ em JP são apresentadas nas Figuras 2.2a e 2.2b com maiores concentrações no período entre o final da manhã e o início da tarde. Nestes períodos existe um grande fluxo de veículos, incluindo os movidos a diesel, consequentemente uma maior emissão de $\mathrm{S}_{2}$. Em relação às estações do ano, outono e inverno, apresentaram as menores concentrações de $\mathrm{SO}_{2}$. Em contrapartida, as concentrações de $\mathrm{S}_{2}$ na TQ, mostrados nas Figuras 2.la e 2.lb apresentaram um comportamento atípico, tendo valores elevados no período noturno.

Uma possível explicação é a movimentação de caminhões de uma empresa de bebidas de grande porte, que possui operação $24 \mathrm{~h}$ de carga e descarga, que pode aumentar a concentração deste poluente pela redução da altura da camada de mistura. Porém apenas está explicação não define de fato o motivo desta anomalia, o que se pode afirmar é que existe uma fonte de emissão de $\mathrm{SO}_{2}$ local.

Foi observado que nas duas EMQAr ocorreram violação ao padrão primário estabelecido para o $0_{3}$. Na TQ ocorreram cinco violações no triênio sendo duas em 2014 (86,4 e 96,2ppb) e três em 2016 (146,3, 102,9 e 109,4 ppb). Em JP ocorreram 307 violações durante o triênio, sendo 212 em 2014, 92 em 2015 e 3 em 2016. Neste caso, como foram muitas violações, as máximas se destacam, sendo 197 ppb em 2014, 173 ppb em 2015 e 95 ppb em 2016. 0 MP10 violou o padrão da qualidade do ar apenas no ano de 2014 com três violações em JP, sendo as duas concentrações máximas de $157 \mu \mathrm{g} \mathrm{m}^{-3}$ e de $153 \mu \mathrm{g} \mathrm{m}^{-3}$.

A Figura 3 apresenta os boxplot para $\mathrm{O}_{3}, \mathrm{NO}_{2}$ e $\mathrm{CO}$ e $\mathrm{MP}_{10}$ e o $\mathrm{SO}_{2}$. 

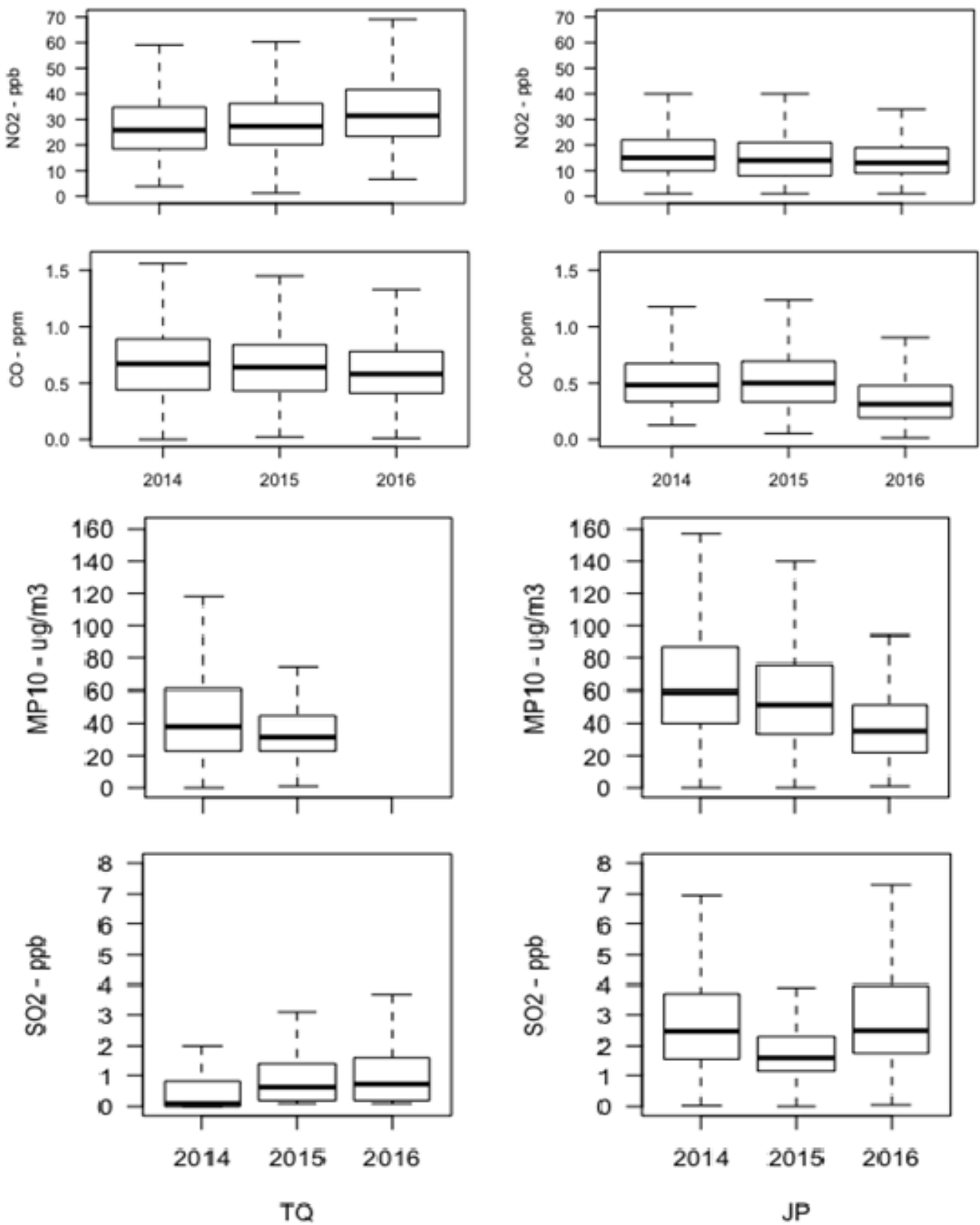

Figura 3 - Boxplot para $0_{3}, \mathrm{NO}_{2}, \mathrm{CO}$ (3.1), $\mathrm{SO}_{2}$ e MP10 (3.2) EMQAr TQ (lado esquerdo) e JP (lado direito).

Fonte: 0s autores

Comparando as duas estações, as maiores concentrações de 03 foram encontradas em JP, assim como o maior número de violações e as concentrações de N02 foram maiores na TQ. As concentrações observadas para o $\mathrm{C} 0$ não apresentam diferença significativa, sugerindo emissões veiculares equivalentes, mesmo os dois locais possuírem diferenças significativas nas emissões totais. As concentrações de MP10 foram maiores em JP assim como as concentrações de S02. A emissão de MP10 e S02 em ambientes urbanos, onde há um baixo índice de indústrias, 
principalmente indústrias de grande porte como refinarias, polos petroquímicos, por exemplo, é predominantemente originária de veículos pesados, que utilizam diesel. A estação de JP apresentou para o período, as maiores concentrações de S02 assim como maiores valores máximos, pois essa estação de qualidade do ar é impactada pelas atividades de uma refinaria, de um polo petroquímico, além de contribuição oriunda de emissão veicular devido ao grande número de veículos a diesel em função das atividades locais e da proximidade de uma rodovia. Interpretação análoga é possível fazer para o MP10 em JP, além da contribuição veicular existe também uma contribuição industrial significativa.

Visando avaliar a influência das variáveis entre si, foi realizada a análise de correlação pelo método de Pearson. As matrizes de correlação, apresentadas na Figura 4 (4.1 e 4.2) comprovam as relações propostas anteriormente. As variáveis NOx e N0 apresentaram alto grau de correlação linear positiva tanto na TQ $(r=0,95)$ quanto em JP $(r=0,97)$, justificado pelo fato do $\mathrm{NOx}$ ser a soma do $\mathrm{NO}$ e $\mathrm{NO}_{2}$.

A relação entre o C0 e o N0x na TQ $(r=0,75)$ e em JP $(r=0,73)$ foram as segundas maiores relações positivas, mostrando que possivelmente ambos os compostos são provenientes de fontes móveis. As maiores relações negativas estão entre temperatura e umidade relativa (TQ, r $=-0,74)$ e $(J P, r=-0,82)$, como esperado.

A correlação entre $0_{3}$ e temperatura é inversamente proporcional em relação a umidade relativa, uma vez que o mesmo se forma em dias mais quentes, que tendem a ser mais secos, e consequentemente possuem menor umidade relativa. A umidade relativa tem influência na solubilização de poluentes hidrossolúveis na forma de aerossóis, causando a deposição destes ou mesmo proporcionando a reação em fase aquosa de forma mais efetiva (GIODA et al., 2018).

A relação negativa entre $\mathrm{O}_{3}$ e $\mathrm{NO}$ é ocasionada pelo fato do $\mathrm{NO}$ reagir com $0 \mathrm{O}_{3}$, formando $\mathrm{NO}_{2}$ e $0_{2}$ como apresentado na reação $1.0 \mathrm{MP}_{10}$ está relacionado positivamente com o $\mathrm{NO}_{2}$ e com o $\mathrm{CO}$, levando a entender que estes podem ser oriundos da mesma fonte de emissão. $0 \mathrm{O}_{3}$ possivelmente possui uma contribuição de transporte com origem nas localidades vizinhas em ambas as estações, em função da dependência deste com a velocidade do vento (0,51 na TQ e 0,50 em JP), porém não é possível afirmar apenas com o teste de correlação. 

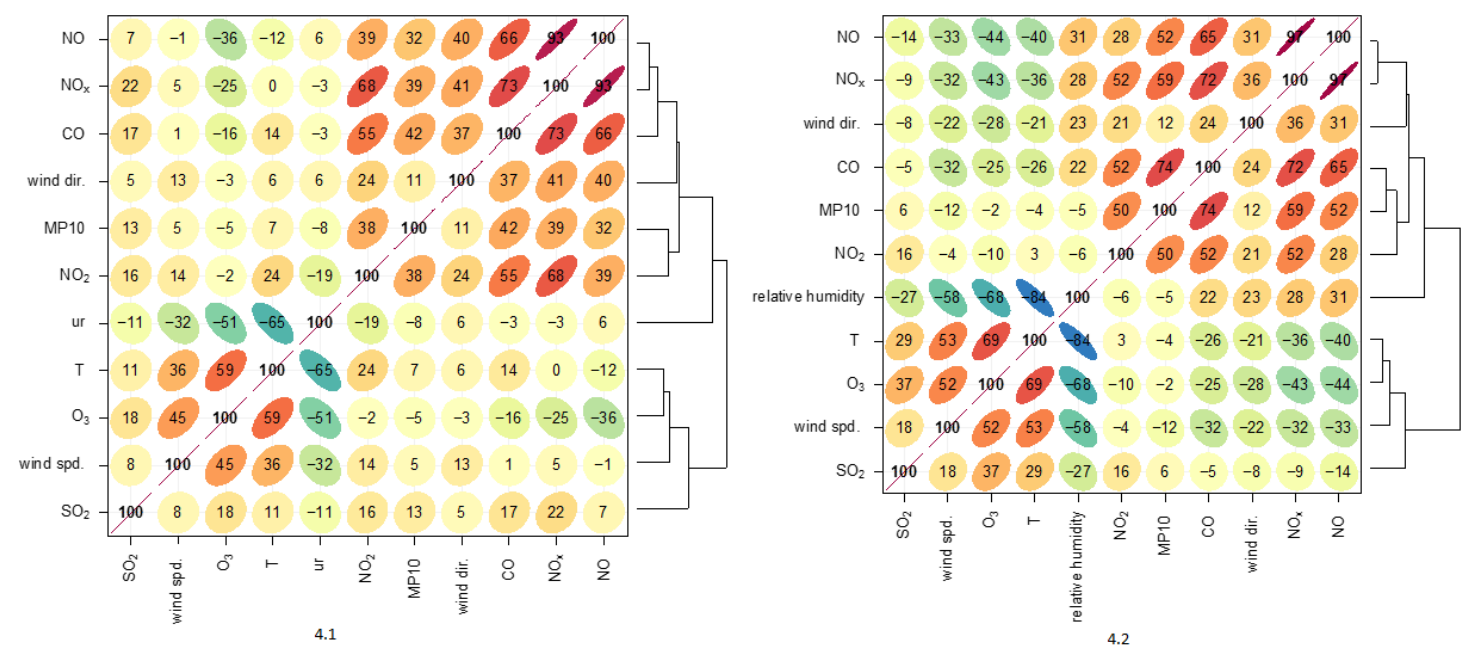

Figura 4 - Matriz de correlação entre todas as variáveis pelo método Pearson para TQ (4.1) e JP (4.2).

Fonte: Os autores

Uma maneira de ver o padrão de um poluente ao longo do dia da semana e em relação a parâmetros meteorológicos de velocidade e direção do vento é a plotagem de gráficos de anéis através da ferramenta polarAnnulus, como mostrado na Figura 5, sendo 5.1 para TQ e 5.2 para JP.
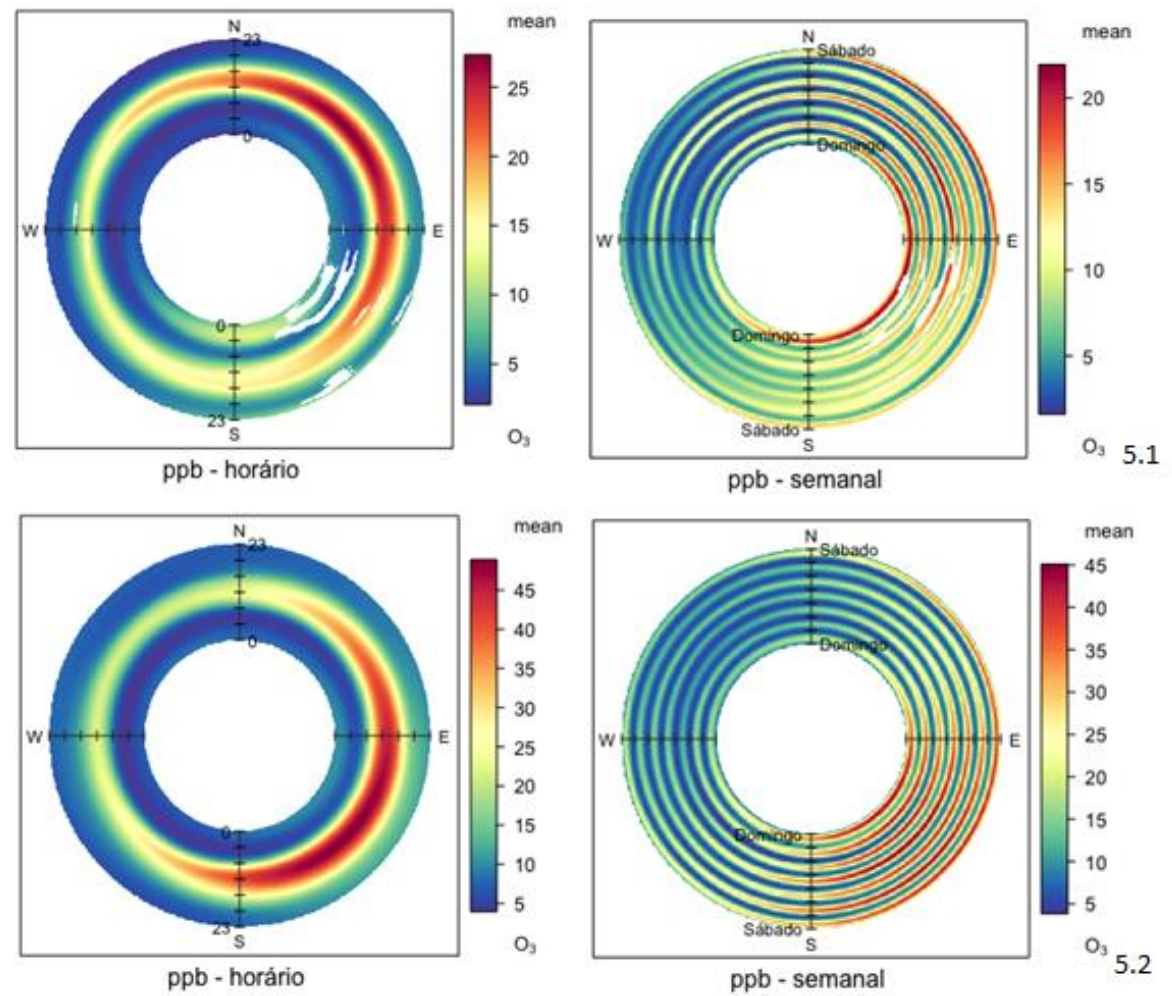

Figura 5 - Distribuição anelar em função das horas e da direção do vento para as concentrações médias horárias de 03 para TQ (5.1) e JP (5.2).

Fonte: Os autores. 
Na TQ, o 03 apresentou maiores concentrações na parte da tarde, entre 12 e 16 h com um grande componente eólico de noroeste, como apresentado na Figura 5.l. Analisando os dias da semana na TQ foi observado altos valores de 03 nos finais de semana, como observado em outros locais da cidade do Rio de Janeiro, como detalhado no estudo de Martins et al. (2015), onde é atribuído à redução da frota circulante de ônibus e caminhões, que são grandes emissores de N0. Com baixa concentração de N0 nos finais de semana há um acúmulo de ozônio troposférico, como observado na TQ. Na Figura 5.2 é possível observar que em JP essa relação não se confirma. Todos os dias da semana apresentaram valores altos de 03 e isto demostra que nesta região a formação de 03 está associada a emissões de seus percursores promovidas por fontes de emissão móveis e fixas. Segundo o (INEA, 2015), esta região vem apresentando altas concentrações de 03, como apresentado nos últimos relatórios de Qualidade do $\operatorname{Ar}$ (2010 a 2015). Os relatórios confirmam a influência das fontes emissoras desta região, principalmente no que tange o denso fluxo de veículos (emissão de NOx) em função da proximidade da rodovia Washington Luiz e as atividades industriais do Polo Petroquímico de Duque de Caxias (emissão de $\mathrm{HC})$.

Comparando-se as reduções em relação a dias de semana e final de semana para NO e as elevações de 03 para o mesmo período, dos resultados encontrados neste estudo com o estudo realizado por Martins et al. (2015) as maiores reduções para as concentrações de N0 durante os finais de semana foram observadas nas estações relativas a este estudo (JP 31 \% e TQ 34 \%) em comparação Bangu (49,9\%), Campo Grande (34,4\%) e Irajá (46,7\%). Para 03, JP foi o local que apresentou o maior aumento durante os finais de semana (35\%), sendo 5,5\% maior que a soma das relações de todas as outras estações (TQ - 12\%; Bangu - 6,8\%; Campo Grande - 3,4\% e Irajá $7,3 \%)$.

Segundo o INEA (2015), os maiores valores de concentração de 03 apresentam relação direta com os maiores valores de concentração dos seus precursores que, em função da exposição à radiação solar e da estabilidade atmosférica, provocam a formação de 03 em concentrações elevadas. No entanto, não é possível deduzir se o aumento dos níveis de ozônio está ligado somente aos seus precursores, uma vez que também pode estar relacionado as condições meteorológicas ou até mesmo do transporte advectivo (direção preferencial dos ventos) de uma região para outra, tendo em vista as complexas interações químicas e meteorológicas envolvidas na formação e transporte deste poluente (MONKS et al.,2015). 


\section{CONCLUSÃ0}

A qualidade do ar em duas regiões distintas (Taquara e Jardim Primavera) dentro da região metropolitana do Rio de Janeiro apresenta comportamentos distintos. Os resultados deste estudo revelam que estações de monitoramento alocadas em ambientes urbanos e industriais apresentarão resultados distintos e que a identificação das fontes emissoras se torna uma tarefa mais complicada em locais onde existem fontes fixas e móveis com grande potencial poluidor. Vale ressaltar que a diferença de relevo e características meteorológicas é pequena. 0 estudo estatístico multivariado aqui apresentado mostra-se uma ferramenta adequada para o entendimento dos dados de qualidade do ar.

Em JP o principal aspecto encontrado foi a diminuição gradativa das concentrações de $0_{3}$ ao longo do triênio, porém mais pela influência das condições locais e do transporte atmosférico do que das elevadas concentrações de seus precursores. Entretanto, existe a possível influência do polo petroquímico de Duque de Caxias na área de abrangência da estação de monitoramento de JP. Para os órgãos ambientais e pesquisadores, o entendimento do comportamento deste poluente no Rio de Janeiro ainda é muito complexo, porém evolui a cada ano com estudos e pesquisas.

Na TQ o principal aumento foi em relação ao $\mathrm{NO}_{2}$. Isto pode estar relacionado ao aumento de ônibus articulados circulantes na região, trocando o cenário de veículos de passeio para veículos pesados, que emitem bem mais NOx e também ao grande fluxo de caminhões no pátio da empresa de distribuição de bebidas situada a frente da estação. Provavelmente, a velocidade média da via deve ter diminuído, porém esta conclusão ficou limitada por falta de dados de fluxos de veículos.

Estudos como aqui apresentados tem sua relevância, uma vez as preocupações quanto aos efeitos adversos que a poluição atmosférica pode causar na saúde humana tem aumento a cada dia. É essencial conhecer como as condições meteorológicas afetam a concentração de ozônio e tentar prever cenários deste poluente é fundamental, dado que pode auxiliar na tomada de decisões das agências ambientais, no que diz respeito ao controle das emissões. 


\section{AGRADECIMENTOS}

Ao INEA pelo fornecimento dos dados das estações de qualidade do ar. Raphael Perciliano da Silva de Almeida - Composição e revisão do artigo, elaboração e execução dos scripts na linguagem R, análise estatística dos resultados. Thainá de Carvalho e Souza - Tratamento dos dados das estações de qualidade do ar, Revisão do artigo, elaboração dos scripts na linguagem R. Simone Lorena Quiterio de Souza - Composição e revisão do artigo, Eduardo Monteiro Martins - Composição e revisão do artigo, formatação e revisão ortográfica. Sergio Machado Corrêa Tratamento dos dados das estações de qualidade do ar, Revisão do artigo, elaboração dos scripts na linguagem $R$.

\section{REFERÊNCIAS BIBLIOGRÁFICAS}

ATKINSON, R. Atmospheric chemistry of VOCs and NO(x). Atmospheric Environment, v. 34, n. 12-14, p. 2063-2101, 2000 .

CARSLAW, D. The openair manual open-source tools for analysing air pollution dataKing's College London, 2015.

CORRÊA, S. M.; MARTINS, E. M.; ARBILLA, G. Formaldehyde and acetaldehyde in a high traffic street of Rio de Janeiro , Brazil. Atmospheric Environment, v. 37, p. 23-29, 2003.

FILIP SOPORAN, V.; NASCUTIU, L.; SOPORAN, B.; PAVAI, C. Case studies of methane dispersion patterns and odor strength in vicinity of municipal solid waste landfill of Cluj-Napoca, Romania, using numerical modeling. Atmospheric Pollution Research, v. 6, n. 2, p. 312-321, 2015. Disponível em: <http://www.sciencedirect.com/science/article/pii/S1309104215302397〉

FUNDAÇ̃O CENTRO ESTADUAL DE ESTATÍSTICAS, PESQUISAS E FORMACÃO DE SERVIDORES PÚBLICOS DO RIO DE JANEIR0 - CEPERJ. Centro de Estatísticas, Estudos e Pesquisas - CEEP, Coordenadoria de Políticas Sociais - COP0S. Análise dos Aspectos Sociais da Qualidade de Vida da População do Estado do Rio de Janeiro, Análise dos Aspectos Sociais da Qualidade de Vida da População do Estado do Rio De Janeiro, Relatório Final, 2018.

GERALDINO, C. G. P.; MARTINS, E. M.; DA SILVA, C. M.; ARBILLA, G. An Analytical Investigation of Ozone Episodes in Bangu, Rio de Janeiro. Bulletin of Environmental Contamination and Toxicology, v. 98, n. 5, p. 632-637, 2017.

GIODA, A.; OLIVEIRA, R. C. G.; CUNHA, C. L.; CORRÊA, S. M. Understanding ozone formation at two islands of Rio de Janeiro, Brazil. Atmospheric Pollution Research, v. 9, n. 2, p. 278-288, 2018. Disponível em: $<$ http://dx.doi.org/10.1016/j.apr.2017.10.003>

IBGE. IBGE | Brasil em Síntese | Rio de Janeiro | Rio de Janeiro | Panorama. 2018. Disponível em: $<$ https://cidades.ibge.gov.br/brasil/rj/rio-de-janeiro/panorama〉. Acesso em: 28 abr. 2019.

INEA. Relatório de Qualidade do Ar do Estado do RIo de Janeiro - Ano base 2015.

MAIA, M.; QUITÉRIO, S. L.; LOPES, R.; LOYOLA, J.; ARBILLA, G. Caracterização de metais traço presentes no material particulado atmosférico inferior a 2,5 $\mathrm{m}\left(\mathrm{MP}_{2,5}\right)$ no entorno de uma área industrial. Perspectivas da Ciência e Tecnologia, v. 6, p. 12, 2014.

MARQUES, L. da C. Modelagem matemática da dispersão de poluentes atmosféricos em indústria siderúrgica no 
Ceará. Dissertação (mestrado) - Universidade Federal do Ceará, Centro de Tecnologia, Programa de Pós-Graduação em Engenharia Civil: Saneamento Ambiental, Fortaleza, 2017, p. 84, 2017.

MARTINS, E. M.; NUNES, A. C. L.; CORRÊA, S. M. Understanding ozone concentrations during weekdays and weekends in the urban area of the city of rio de janeiro. Journal of the Brazilian Chemical Society, v. 26, n. 10, p. 1967-1975, 2015.

MICHANOWICZ, D. R.; SHMOOL, J. L. C.; TUNNO, B. J.; TRIPATHY, S.; GILLOOLY, S.; KINNEE, E.; CLOUGHERTY, J. E. A hybrid land use regression/AERMOD model for predicting intra-urban variation in PM2.5. Atmospheric Environment, v. 131, p. 307-315, 2016. Disponível em: 〈http://dx.doi.org/10.1016/j.atmosenv.2016.01.045>

MONKS, P. S.; ARCHIBALD, A. T.; COLETTE, A.; COOPER, 0.; COYLE, M.; DERWENT, R.; FOWLER, D.; GRANIER, C.; LAW, K. S.; MILLS, G. E.; STEVENSON, D. S.; TARASOVA, 0.; THOURET, V.; VON SCHNEIDEMESSER, E.; SOMMARIVA, R.; WILD, 0.; WILLIAMS, M. L. Tropospheric ozone and its precursors from the urban to the global scale from air quality to shortlived climate forcer. Atmospheric Chemistry and Physics, v. 15, n. 15, p. 8889-8973, 2015.

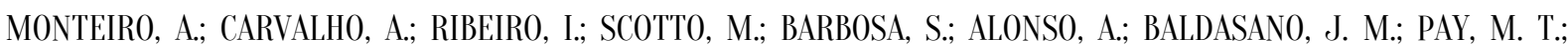
MIRANDA, A. I.; BORREG0, C. Trends in ozone concentrations in the Iberian Peninsula by quantile regression and clustering. Atmospheric Environment, v. 56, p. 184-193, 2012. Disponivel em: 〈http://dx.doi.org/10.1016/j.atmosenv.2012.03.069>

MOREIRA, D.; TIRABASSI, T. Modelo matemático de dispersão de poluentes na atmosfera: um instrumento técnico para a gestão ambiental. Ambiente \& Sociedade, v. 7, n. 2, p. 159-172, 2006.

MUNIR, S.; CHEN, H.; ROPKINS, K. Quantifying temporal trends in ground level ozone concentration in the UK. Science of the Total Environment, v. 458-460, p. 217-227, 2013. Disponivel em: <http://dx.doi.org/10.1016/j.scitotenv.2013.04.045>

NETO, R. P. D. M. Dianóstico ambiental sobre material particulado em suspensão no entorno de pedreira: um estudo de caso em Jaboatão do Guararapes/PE. Dissertação (Mestrado) - Universidade Federal de Pernambuco. CTG. Programa de Pós-Graduação em Engenharia Mineral, 2012, p. 111, 2012.

ORLANDO, J. P.; ALVIM, D. S.; YAMAZAKI, A.; CORRÊA, S. M.; GATTI, L. V. Ozone precursors for the São Paulo Metropolitan Area. Science of the Total Environment, v. 408, n. 7, p. 1612-1620, 2010. Disponível em: $\langle$ http://dx.doi.org/10.1016/j.scitotenv.2009.11.060>

R CORE TEAM. CRAN Task Views. 2017. Disponível em: <https://cran.r-project.org/web/views/>. Acesso em: 26 jul. 2017.

UEDA, A. C. Estudo de Compostos Orgânicos Voláteis na Atmosfera da Região Metropolitana de Campinas. Tese de Doutorado - Universidade Estadual de Campinas, Faculdade de Engenharia Química, p. 256, 2010.

VALOTTO, G.; VARIN, C. Characterization of hourly N0x atmospheric concentrations near the Venice International Airport with additive semi-parametric statistical models. Atmospheric Research, v. 167, n. x, p. 216-223, 2016. Disponivel em: 〈http://dx.doi.org/10.1016/j.atmosres.2015.07.023〉

VITALI, L.; MORABITO, A.; ADANI, M.; ASSENNAT0, G.; CIANCARELLA, L.; CREMONA, G.; GIUA, R.; PASTORE, T.; PIERSANTI, A.; RIGHINI, G.; RUSSO, F.; SPAGNOLO, S.; TANZARELLA, A.; TINARELLI, G.; ZANINI, G. A Lagrangian modelling approach to assess the representativeness area of an industrial air quality monitoring station. Atmospheric Pollution Research, v. 7, n. 6, p. 990-1003, 2016. Disponível em: 〈http://dx.doi.org/10.1016/j.apr.2016.06.002>

WANG, F.; CHEN, D. S.; CHENG, S. Y.; LI, J. B.; LI, M. J.; REN, Z. H. Identification of regional atmospheric PM10 transport pathways using HYSPLIT, MM5-CMAQ and synoptic pressure pattern analysis. Environmental Modelling and Software, v. 25, n. 8, p. 927-934, 2010. Disponivel em: 〈http://dx.doi.org/10.1016/j.envsoft.2010.02.004〉. 